\title{
Computation of Greeks and Multidimensional Density Estimation for Asset Price Models with Time-Changed Brownian Motion
}

\author{
REIICHIRO KAWAI* AND ARtURO KoHATSU-HigA ${ }^{\dagger}$
}

\begin{abstract}
The main purpose of this paper is to propose computational methods for Greeks and the multidimensional density estimation for an asset price dynamics model defined with time-changed Brownian motions. Our approach is based on an application of the Malliavin integration-by-parts formula on the Gaussian space conditioning on the jump component. Some numerical examples are presented to illustrate the effectiveness of our results.

Keywords: Integration-by-parts formula, Malliavin calculus, normal inverse Gaussian process, time-changed Brownian motion, variance gamma process.

2000 Mathematics Subject Classification: 60H07, 60E07, 60G51, 91B70
\end{abstract}

\section{Introduction}

The Malliavin integration-by-parts formula was established in the historic paper of Bismut [5], and served as a key tool to reveal the relation between the Hörmander condition on the hypoelliptic problem and the existence of smooth densities for solutions to stochastic differential equations. Moreover, in his book [6], he studied the logarithmic gradient of the fundamental solution to the heat equation on a Riemaniann manifold. In mathematical finance, Malliavin calculus on the Wiener space is applied in Fournié et al. [15] to the sensitivity analysis for the Black-Scholes asset price dynamics model. Moreover, the so-called Malliavin-Thalmaier integration-by-parts formula was introduced in Malliavin and Thalmaier [21] with a view towards the computation of multidimensional probability density functions, of which numerical issues are studied in Kohatsu-Higa and Yasuda [19]. For stochastic differential equations driven by Brownian motion, the technique based on the integration-by-parts formula from Malliavin Calculus has become standard.

It is then a natural question whether a similar approach can be obtained in the case of computation of Greeks and the multidimensional density for jump processes. Concerning Malliavin calculus for jump processes, the existence of weights for the logarithmic gradient dates back to the work of Bichteler, Gravereaux, Jacod [4]. For practical considerations, it is important to derive explicit weights, in order to design an efficient Monte Carlo evaluation. In particular, Davis and Johansson [12], and Cass and Friz [10] studied the Malliavin Greeks for jump diffusion processes. Their approach consists of conditioning by the jump component and then performing the Malliavin calculus techniques with respect to the Brownian motion component. In other words, their models are required to be a superposition of independent jump and diffusion components, where the diffusion must be nondegenerate. On the other hand, ElKhatib and Privault [13] applied the calculus focused on the Poisson arrival times of Carlen and Pardoux [7], while Bally et al. [1] took a unified approach considering the derivatives with respect to both the Poisson arrival times and the amplitudes of the jumps. Takeuchi studied in [27] the same problem of solutions to stochastic differential equations with jumps via the martingale approach, without using the approach introduced in [4]. Kawai and Takeuchi [17, 18] derive Greeks formulas for asset price dynamics described by gamma processes, based upon a scaling property of gamma processes with respect to the Esscher transform parameter. It seems that there is still no unified approach to the sensitivity analysis and also the multidimensional density estimation for random processes beyond the pure diffusion cases.

\footnotetext{
Published in Applied Mathematical Finance (2010) 17(4) 301-321.

*Email address: reiichiro.kawai@gmail.com. Postal address: Department of Mathematics, University of Leicester, Leicester LE1 7RH, UK.

${ }^{\dagger}$ Email address: kohatsu@sigmath.es.osaka-u.ac.jp. Postal address: Graduate School of Engineering Sciences, Osaka University, 560-8531, Japan.
} 
The main purpose of this paper is to obtain an expression for the calculation of Greek and the multidimensional density for an asset price dynamics model defined with time-changed Brownian motions. As will be illustrated later, our framework encompasses some Lévy process models and stochastic volatility models. Our approach is based on an application of the Malliavin integration-by-parts formula on the Gaussian space conditioning on the jump component. We consider this approach best suited for actual financial models as we reflect in the examples that we have considered. This approach has been taken in [10, 12]. Nevertheless they use the representation of the model as a stochastic differential equation while we use an expression as subordinated Wiener processes. In the Lévy processes framework with an expression as a time-changed Brownian motion, our results improve those of [1, 13] in the sense that our Lévy processes are of more realistic infinite activity type. When restricted to the variance gamma model, our results can be recovered from those of [17, 18].

The rest of this paper is organized as follows. We first introduce in Section 2 the time-changed Brownian motion and give three examples of time-changed Brownian motions of practical interest. In Section 3 , we apply the Malliavin integration-by-parts formula on the Gaussian space to derive unbiased estimators of various Greeks in our model setting with a view towards efficient Monte Carlo evaluation. We next present in Section 4 that the Malliavin-Thalmaier integration-by-parts formula can also be applied to the multidimensional density estimation in our framework. Finally, Section 5 concludes.

\section{Preliminaries}

Let us begin with general notations which will be used throughout the text. We let $\mathbb{N}$ be the set of positive integers. We denote by $\mathbb{R}^{d}$ the $d$-dimensional Euclidean space with the norm $\|\cdot\|$ and the inner product by $\langle\cdot, \cdot\rangle$. We fix $(\Omega, \mathscr{F}, \mathbb{P})$ as our underlying probability space. We mean by the time-changed Brownian motion the following stochastic process

$$
X_{t}:=\mu t+\theta Y_{t}+\sigma W_{Y_{t}},
$$

where $\mu \in \mathbb{R}, \theta \in \mathbb{R}, \sigma>0$, and $\left\{W_{t}: t \geq 0\right\}$ is a standard Brownian motion in $\mathbb{R}$, and $\left\{Y_{t}: t \geq 0\right\}$ is a non-decreasing stochastic process in $\mathbb{R}$, with $Y_{0}=0$, a.s., independent of $\left\{W_{t}: t \geq 0\right\}$. For convenience, we define the characteristic exponent

$$
e^{\varphi_{Y_{t}}(y)}:=\mathbb{E}\left[e^{i y Y_{t}}\right] .
$$

Intuitively, one may regard the original clock $\{t: t \geq 0\}$ as calendar time, while the new random clock $\left\{Y_{t}: t \geq 0\right\}$ as business time. A more active business day implies a faster business clock, and vice versa.

\subsection{Examples of Time-Changed Brownian Motion}

If $\left\{Y_{t}: t \geq 0\right\}$ is a subordinator, that is, an one-sided Lévy process, the above time-changed Brownian motion is a Lévy process, which is often called Lévy process of type G. (See, for example, Rosiński [24].) Moreover, unless it is a deterministic subordinator (that is, $Y_{t}=a t, a>0$ ), the time-changed Brownian motion becomes a pure-jump Lévy process. In Example 2.1 and 2.2, we give two such examples.

Example 2.1. (Variance gamma Lévy processes) The stochastic process (2.1) is called a variance gamma (Lévy) process of Madan, Carr and Chang [20], if we set $\left\{Y_{t}: t \geq 0\right\}$ to be a gamma process, whose characteristic exponent is given by $\varphi_{Y_{t}}(y)=-a t \ln (1-i y / b)$, while its marginal density is given in closed form by

$$
f_{Y_{t}}(x)=\frac{b^{a t}}{\Gamma(a t)} x^{a t-1} e^{-b x}, \quad x>0,
$$

with $a=b=: v^{-1}>0$. The model has attracted the attention of finance practitioners, and thus appears often in the computational finance literature (see for example, Carr and Madan [9] and Fu [16]). The characteristic function of $X_{t}=\mu t+\theta Y_{t}+\sigma W_{Y_{t}}$ is given by

$$
\mathbb{E}\left[e^{i y X_{t}}\right]=e^{i y \mu t}\left(1-i y \theta v+\frac{1}{2} \sigma^{2} v y^{2}\right)^{-t / v} .
$$

Moreover, it is also shown in [20] that the variance gamma process can be expressed as the difference of two independent gamma processes,

$$
X_{t}=Y_{t, p}-Y_{t, n},
$$


where the gamma processes $\left\{Y_{t, p}: t \geq 0\right\}$ and $\left\{Y_{t, n}: t \geq 0\right\}$ can be characterized by (2.2) with $(a, b)=\left(v^{-1},\left(\mu_{p} v\right)^{-1}\right)$ and $(a, b)=\left(v^{-1},\left(\mu_{n} v\right)^{-1}\right)$ for some $v>0$, where the parameters are given by $\mu_{p}=\frac{1}{2} \sqrt{\theta^{2}+2 \sigma^{2} / v}+\theta / 2$ and $\mu_{n}=\frac{1}{2} \sqrt{\theta^{2}+2 \sigma^{2} / v}-\theta / 2$, respectively.

Example 2.2. (Normal inverse Gaussian Lévy processes) The normal inverse Gaussian process can be expressed as a time-changed Brownian motion by setting $\left\{Y_{t}: t \geq 0\right\}$ to be a Lévy process induced by the inverse Gaussian distribution, whose marginal at time $t$ is identical in law to the first time that a Brownian motion with drift $v$ reaches the positive level $t$, that is,

$$
\mathbb{E}\left[e^{y Y_{t}}\right]=\exp \left[-t\left(\sqrt{-2 y+v^{2}}-v\right)\right] .
$$

Then, the normal inverse Gaussian process $X_{t}=\mu t+\theta Y_{t}+\sigma W_{Y_{t}}$ has the characteristic function

$$
\mathbb{E}\left[e^{i y X_{t}}\right]=e^{i y \mu t} \exp \left[-t \sigma\left(\sqrt{\frac{v^{2}}{\sigma^{2}}+\frac{\theta^{2}}{\sigma^{4}}-\left(\frac{\theta}{\sigma^{2}}+i y\right)^{2}}-\frac{v}{\sigma}\right)\right],
$$

where $v>0$. Conversely, set $\alpha=\sqrt{v^{2} / \sigma^{2}+\theta^{2} / \sigma^{4}}, \beta=\theta / \sigma^{2}$, and $\delta=\sigma$. Then, we get $|\beta|<\alpha, v=\delta \sqrt{\alpha^{2}-\beta^{2}}$, and

$$
X_{t}=\mu t+\beta \delta^{2} Y_{t}+\delta W_{Y_{t}} .
$$

See Barndorff-Nielsen [3], Carr et al.[8] and Schoutens [26] for more details.

It has been a general consensus that return volatilities vary stochastically over time. The concept of "time change" can also be applied to capture the evidence of stochastic volatility. That is, by the Gaussian scaling property, random changes in volatility can be related to random time changes. The following is an illustrative example of the business clock, which is successfully applied in Carr et al.[8] to control the clock of pure-jump Lévy processes.

Example 2.3. (Brownian motion, time changed via integrated Cox-Ingersoll-Ross (CIR) square-root process) Let $\left\{z_{t}: t \geq 0\right\}$ be a mean-reverting positive process defined via the stochastic differential equation

$$
d z_{t}=\kappa\left(\eta-z_{t}\right) d t+\lambda \sqrt{z_{t}} d W_{t},
$$

where $z_{0}, \kappa, \eta, \sigma$ are positive constants with $2 \kappa \eta>\sigma^{2}$. The parameter $\eta$ serves as the long run rate of time change, $\kappa$ is the mean reversion rate, while $\lambda$ is the volatility of the time change. This is often called the CIR square root process [11]. Taking the process $\left\{z_{t}: t \geq 0\right\}$ as the instantaneous rate of time change, we define an increasing process $Y_{t}:=\int_{0}^{t} z_{s} d s$. It is well known that the characteristic function of $Y_{t}$ is given by

$$
\phi_{t}(y):=\mathbb{E}\left[e^{i y Y_{t}}\right]=e^{A(t, y) z_{0}} B(t, y),
$$

where $\gamma:=\sqrt{\kappa^{2}-2 \lambda^{2} i y}, A(t, y):=2 i y /(\kappa+\gamma \operatorname{coth}(\gamma t) / 2)$, and

$$
B(t, y):=\exp \left[\kappa^{2} \eta t / \lambda^{2}\right]\left(\cosh \left(\frac{\gamma t}{2}\right)+\frac{\kappa}{\gamma} \sinh \left(\frac{\gamma t}{2}\right)\right)^{-2 \kappa \eta / \lambda^{2}} .
$$

Using the above fact, the characteristic function of $X_{t}=\mu t+\theta Y_{t}+\sigma W_{Y_{t}}$ can be derived as

$$
\mathbb{E}\left[e^{i y X_{t}}\right]=e^{i y \mu t} \mathbb{E}\left[e^{\left(i y \theta-y^{2} \sigma^{2} / 2\right) Y_{t}}\right]=e^{i y \mu t} \phi_{t}\left(y \theta+i y^{2} \sigma^{2} / 2\right),
$$

where the computation is performed given $Y_{t}$. For more details, we refer, for example, to Carr et al.[8] and Schoutens [26].

\subsection{Malliavin Calculus. Some notations and properties}

Let us next present some basic facts on the Malliavin calculus. For details and notation, we refer to the book of Nualart [23]. Fix $T>0, d_{1} \in \mathbb{N}, d_{2} \in \mathbb{N}$, and let $\left\{W_{t}: t \geq 0\right\}$ be a standard Brownian motion in $\mathbb{R}^{d_{1}}$, independent of $\left\{Y_{t}: t \in[0, T]\right\}$, with $W_{t}:=\left(W_{t}^{(1)}, \ldots, W_{t}^{\left(d_{1}\right)}\right)^{\prime}$. Throughout this study, the time-changing process $\left\{Y_{t}: t \in[0, T]\right\}$ is one-dimensional. Let $\left(\mathscr{F}_{t}\right)_{t \in[0, T]}$ and $\left(\mathscr{G}_{t}\right)_{t \geq 0}$ be the natural filtrations generated respectively by $\left\{Y_{t}: t \in[0, T]\right\}$ and 
$\left\{W_{Y_{t}}: t \geq 0\right\}$. Let $F:=\left(F_{1}, \ldots, F_{d_{2}}\right)^{\prime}$ be a random vector in $\mathbb{R}^{d_{2}}$, measurable with respect to $\mathscr{G}_{T}:=\sigma\left(W_{Y_{t}} ; t \in[0, T]\right)$. We denote by $D^{(k)}$ the Malliavin derivative on the Gaussian space, conditionally on the time changing process, with respect to the $k$-th component of the underlying Brownian motion. Define for each $k=1, \ldots, d_{2}$,

$$
D_{s} F_{k}:=\left[D_{s}^{(1)} F_{k}, \ldots, D_{s}^{\left(d_{1}\right)} F_{k}\right]^{\prime} \text {. }
$$

Moreover, we denote by $\delta_{k}$ the Skorohod integral over $\left(0, Y_{T}\right]$ given $\mathscr{F}_{T}$ with respect to the $k$-th component of the underlying Brownian motion, that is, for a suitable smooth stochastic process $\left\{G_{t}: t \geq 0\right\}$ in $\mathbb{R}^{d_{2}}$,

$$
\delta_{k}(G .):=\int_{0}^{Y_{T}} G_{s} \delta W_{s}^{(k)},
$$

where the multivariate Skorohod integral is taken componentwise. Both the operators $D$ and $\delta$ are linked by the equality; for each $k=1, \ldots, d_{2}$,

$$
\delta_{k}(\langle F, G .\rangle)=\left\langle\delta_{k}(G .), F\right\rangle-\int_{0}^{Y_{T}}\left\langle D_{t}^{(k)} F, G_{t}\right\rangle d t .
$$

Moreover, taking the conditional expectation, we get the duality; for each $k=1, \ldots, d_{2}$,

$$
\mathbb{E}\left[\int_{0}^{Y_{T}}\left\langle D_{t}^{(k)} F, G_{t}\right\rangle d t \mid \mathscr{F}_{T}\right]=\mathbb{E}\left[\left\langle\delta_{k}(G .), F\right\rangle \mid \mathscr{F}_{T}\right] .
$$

Here, we have assumed that $F \in \mathbb{D}^{1,2}$ and $G \in \operatorname{Dom}(\delta)$.

We next give an integration by parts formula in one dimension (the multidimensional case is introduced in Section 4), which is our key tool to integrate the derivative in the following expectation for $\Phi \in C_{b}^{1}(\mathbb{R} ; \mathbb{R})$,

$$
\mathbb{E}\left[\Phi^{\prime}(X) Z \mid \mathscr{F}_{T}\right]=\mathbb{E}\left[\Phi(X) H \mid \mathscr{F}_{T}\right],
$$

where $X, Z$ and $H$ are suitable random variables. We follow the argument of Montero and Kohatsu-Higa [22]. By the chain rule of the Malliavin derivative, we have that given $\mathscr{F}_{T}$ and for $t \in\left[0, Y_{T}\right]$,

$$
\left(D_{t} \Phi(X)\right) Z h_{t}=\Phi^{\prime}(X) Z h_{t} D_{t} X,
$$

where $\left\{h_{t}: t \in\left[0, Y_{T}\right]\right\}$ is a suitable "smooth" stochastic process such that the terms appearing below are well defined. Integration over $\left[0, Y_{T}\right]$ leads to

$$
\Phi^{\prime}(X) Z=\int_{0}^{Y_{T}} D_{t} \Phi(X) \frac{Z h_{t}}{\int_{0}^{Y_{T}} h_{s} D_{s} X d s} d t
$$

Using the duality (2.4), we get

$$
\mathbb{E}\left[\Phi^{\prime}(X) Z \mid \mathscr{F}_{T}\right]=\mathbb{E}\left[\int_{0}^{Y_{T}} D_{t} \Phi(X) \frac{Z h_{t}}{\int_{0}^{Y_{T}} h_{s} D_{S} X d s} d t \mid \mathscr{F}_{T}\right]=\mathbb{E}\left[\Phi(X) \delta\left(\frac{Z h .}{\int_{0}^{Y_{T}} h_{s} D_{S} X d s}\right) \mid \mathscr{F}_{T}\right] .
$$

We will often refer this relation to the (conditional) integration-by-parts formula in what follows.

\section{Computation of Greeks}

Fix $T>0$ and assume that $\left\{Y_{t}: t \in[0, T]\right\}$ is such that

$$
\left|\varphi_{Y_{t}}\left(-i\left(\theta+\sigma^{2} / 2\right)\right)\right|<+\infty, \quad t \in[0, T] .
$$

We first consider an one-dimensional asset dynamics driven by a time-changed Brownian motion,

$$
S_{t}:=S_{0} e^{r t} \frac{e^{X_{t}}}{\mathbb{E}\left[e^{X_{t}}\right]}=S_{0} e^{r t} \exp \left[\theta Y_{t}+\sigma W_{Y_{t}}-\varphi_{Y_{t}}\left(-i\left(\theta+\sigma^{2} / 2\right)\right)\right],
$$

where the expectation is henceforth taken with respect to a risk neutral probability. With $\left\{Y_{t}: t \in[0, T]\right\}$ in Example 2.1, 2.2 and 2.3 the discounted stock price model (3.2) is clearly a martingale with respect to the risk neutral measure under the integrability condition (3.1) due to the Markovian property of $\left\{Y_{t}: t \in[0, T]\right\}$.

If the business clock $\left\{Y_{t}: t \in[0, T]\right\}$ is deterministic, then our framework simply reduces to the most basic BlackScholes model. To avoid such triviality, we rule out the deterministic clock a priori. Until Section 3.5, we work on the above one-dimensional model, and thus suppress the scripts in the Malliavin derivative $D^{(k)}$ and in the Skorohod integral $\delta_{k}$. 


\subsection{Delta}

We first derive an unbiased estimator of the so-called delta, which is the sensitivity index with respect to the initial value.

Proposition 3.1. Assume that $\mathbb{E}\left[S_{T}^{2}\right]<+\infty$. Let $\Phi: \mathbb{R} \rightarrow \mathbb{R}$ be a measurable function such that $\mathbb{E}\left[\Phi\left(S_{T}\right)^{2}\right]$ is locally uniformly bounded in $S_{0}>0$. Moreover, assume that $\mathbb{E}\left[Y_{T}^{-2}\right]<+\infty$. Then, we have

$$
\frac{\partial}{\partial S_{0}} \mathbb{E}\left[\Phi\left(S_{T}\right)\right]=\frac{1}{\sigma S_{0}} \mathbb{E}\left[\Phi\left(S_{T}\right) \frac{W_{Y_{T}}}{Y_{T}}\right] .
$$

Proof. First, assume $\Phi \in C_{b}^{2}(\mathbb{R} ; \mathbb{R})$. We have

$$
\frac{\partial}{\partial S_{0}} \mathbb{E}\left[\Phi\left(S_{T}\right)\right]=\mathbb{E}\left[\frac{\partial}{\partial S_{0}} \Phi\left(S_{T}\right)\right]=\mathbb{E}\left[\Phi^{\prime}\left(S_{T}\right) \frac{\partial S_{T}}{\partial S_{0}}\right]=S_{0}^{-1} \mathbb{E}\left[\Phi^{\prime}\left(S_{T}\right) S_{T}\right]
$$

where the interchange of the derivative and the expectation is justified by the dominated convergence theorem. In fact, as $\varepsilon \downarrow 0$,

$$
\frac{\Phi\left(\left(1+\varepsilon / S_{0}\right) S_{T}\right)-\Phi\left(S_{T}\right)}{\varepsilon} \rightarrow \Phi^{\prime}\left(S_{T}\right) \frac{S_{T}}{S_{0}}, \quad \text { a.s. }
$$

and by the Taylor theorem,

$$
\left|\frac{\mathbb{E}\left[\Phi\left(\left(1+\varepsilon / S_{0}\right) S_{T}\right)-\Phi\left(S_{T}\right)\right]}{\varepsilon}\right| \leq \int_{0}^{1} \mathbb{E}\left[\left|\Phi^{\prime}\left(\left(1+\delta \varepsilon / S_{0}\right) S_{T}\right) \frac{\left(1+\delta \varepsilon / S_{0}\right) S_{T}}{S_{0}}\right|\right] d \delta,
$$

which is clearly uniformly bounded in $\varepsilon$.

Next, given $\mathscr{F}_{T}$, we can apply the conditional integration-by-parts formula (2.5) with $h_{t} \equiv 1$ as

$$
\mathbb{E}\left[\Phi^{\prime}\left(S_{T}\right) S_{T} \mid \mathscr{F}_{T}\right]=\mathbb{E}\left[\Phi\left(S_{T}\right) \delta\left(\frac{S_{T}}{\int_{0}^{Y_{T}} D_{S} S_{T} d s}\right) \mid \mathscr{F}_{T}\right]=\mathbb{E}\left[\Phi\left(S_{T}\right) \frac{W_{Y_{T}}}{\sigma Y_{T}} \mid \mathscr{F}_{T}\right],
$$

where the last equality holds since given $\mathscr{F}_{T}$,

$$
\int_{0}^{Y_{T}} D_{s} S_{T} d s=\sigma S_{T} \int_{0}^{Y_{T}} \mathbb{1}\left(s \leq Y_{T}\right) d s=\sigma S_{T} Y_{T}
$$

Taking the expectation on the both sides, we get the desired result for $\Phi \in C_{b}^{2}(\mathbb{R} ; \mathbb{R})$.

It remains to remove the regularity assumption on $\Phi$. To this end, let us come back to $\Phi$ such that $\mathbb{E}\left[\Phi\left(S_{T}\right)^{2}\right]$ is locally uniformly bounded in $S_{0}$. We can always find a sequence $\left\{\Phi_{n}\right\}_{n \in \mathbb{N}}$ of continuously differentiable functions, from $\mathbb{R}$ to $\mathbb{R}$, with compact support such that $\lim _{n \uparrow+\infty} \mathbb{E}\left[\left|\Phi_{n}\left(S_{T}\right)-\Phi\left(S_{T}\right)\right|^{2}\right]=0$. Hence, by the Cauchy-Schwartz inequality with $\mathbb{E}\left[\left|W_{Y_{T}} / Y_{T}\right|^{2}\right]=\mathbb{E}\left[Y_{T}^{-1}\right]<+\infty$, we have that for each $S_{0}$,

$$
\left|\mathbb{E}\left[\Phi_{n}\left(S_{T}\right) \frac{W_{Y_{T}}}{Y_{T}}-\Phi\left(S_{T}\right) \frac{W_{Y_{T}}}{Y_{T}}\right]\right|^{2} \leq \mathbb{E}\left[\left|\Phi_{n}\left(S_{T}\right)-\Phi\left(S_{T}\right)\right|^{2}\right] \mathbb{E}\left[\left|\frac{W_{Y_{T}}}{Y_{T}}\right|^{2}\right] \rightarrow 0,
$$

as $n \uparrow+\infty$. Then, we obtain that

$$
\mathbb{E}\left[\Phi_{n}\left(\left(1+\varepsilon / S_{0}\right) S_{T}\right)\right]-\mathbb{E}\left[\Phi_{n}\left(S_{T}\right)\right]=\int_{0}^{\varepsilon} \mathbb{E}\left[\Phi_{n}\left(\left(1+h / S_{0}\right) S_{T}\right) \frac{W_{Y_{T}}}{Y_{T}}\right] d h .
$$

Then, by taking limits with respect to $n$, we obtain that $\mathbb{E}\left[\Phi\left(\left(1+\varepsilon / S_{0}\right) S_{T}\right)\right]$ is continuous in $S_{0}$. In a similar fashion, we can prove that $\mathbb{E}\left[\Phi\left(S_{T}\right) W_{Y_{T}} / Y_{T}\right]$ is continuous in $S_{0}$. Finally, by taking limits with respect to $n$ in (3.5) and dividing by $\varepsilon$, we obtain that $\mathbb{E}\left[\Phi\left(S_{T}\right)\right]$ is differentiable with respect to $S_{0}$ and that the desired formula holds. The proof is complete. 
Remark 3.2. The sensitivity with respect to the risk-free rate $r$ and the drift parameter $\theta$ can also be derived via the identity (3.3), respectively as

$$
\frac{\partial}{\partial r} \mathbb{E}\left[\Phi\left(S_{T}\right)\right]=\mathbb{E}\left[\Phi^{\prime}\left(S_{T}\right) \frac{\partial}{\partial r} S_{T}\right]=T \mathbb{E}\left[\Phi^{\prime}\left(S_{T}\right) S_{T}\right]=S_{0} T \frac{\partial}{\partial S_{0}} \mathbb{E}\left[\Phi\left(S_{T}\right)\right]
$$

and

$$
\frac{\partial}{\partial \theta} \mathbb{E}\left[\Phi\left(S_{T}\right)\right]=\mathbb{E}\left[\Phi^{\prime}\left(S_{T}\right) \frac{\partial}{\partial \theta} S_{T}\right]=\mathbb{E}\left[\mathbb{E}\left[\Phi^{\prime}\left(S_{T}\right) S_{T} \mid \mathscr{F}_{T}\right] Y_{T}\right]=\frac{1}{\sigma} \mathbb{E}\left[\Phi\left(S_{T}\right) W_{Y_{T}}\right]
$$

where the interchanges of the derivative and the expectation can be justified as before. Moreover, note that the proof of Proposition 3.1 does not require the Markovian property of the time-changing process $\left\{Y_{t}: t \in[0, T]\right\}$.

\subsection{Vega}

In our framework, we may naturally regard the parameter $\sigma$ as the volatility. The next formula is the so-called vega, which is the sensitivity of the premium with respect to the volatility parameter.

Proposition 3.3. Assume $\mathbb{E}\left[S_{T}^{2} W_{Y_{T}}^{2}\right]<+\infty$. Let $\Phi: \mathbb{R} \rightarrow \mathbb{R}$ be a measurable function such that $\mathbb{E}\left[\Phi\left(S_{T}\right)^{2}\right]$ is locally uniformly bounded in $S_{0}>0$. Assume further that $\mathbb{E}\left[Y_{T}^{-2}\right]<+\infty$. Then,

$$
\frac{\partial}{\partial \sigma} \mathbb{E}\left[\Phi\left(S_{T}\right)\right]=\frac{1}{\sigma} \mathbb{E}\left[\Phi\left(S_{T}\right)\left(\frac{W_{Y_{T}}^{2}}{Y_{T}}-\frac{W_{Y_{T}}}{Y_{T}} \frac{\partial}{\partial \sigma} \varphi_{Y_{T}}\left(-i\left(\theta+\sigma^{2} / 2\right)\right)-1\right)\right]
$$

Proof. As before, it suffices to show the result with $\Phi \in C_{b}^{2}(\mathbb{R} ; \mathbb{R})$. We have

$$
\frac{\partial}{\partial \sigma} \mathbb{E}\left[\Phi\left(S_{T}\right)\right]=\mathbb{E}\left[\Phi^{\prime}\left(S_{T}\right) \frac{\partial S_{T}}{\partial \sigma}\right]=\mathbb{E}\left[\Phi^{\prime}\left(S_{T}\right) S_{T}\left(W_{Y_{T}}-\frac{\partial}{\partial \sigma} \varphi_{Y_{T}}\left(-i\left(\theta+\sigma^{2} / 2\right)\right)\right)\right],
$$

where the interchange of the derivative and the expectation can be justified by the dominated convergence theorem in a similar manner to the proof of Proposition 3.1 .

Next, in view of the delta formula in Proposition 3.1 , it remains to work on $\mathbb{E}\left[\Phi^{\prime}\left(S_{T}\right) S_{T} W_{Y_{T}}\right]$. Given $\mathscr{F}_{T}$, we apply the conditional integration-by-parts formula (2.5) with $h_{t} \equiv 1$ as

$$
\mathbb{E}\left[\Phi^{\prime}\left(S_{T}\right) S_{T} W_{Y_{T}}\right]=\mathbb{E}\left[\mathbb{E}\left[\Phi\left(S_{T}\right) \delta\left(\frac{S_{T} W_{Y_{T}}}{\int_{0}^{Y_{T}} D_{u} S_{T} d u}\right) \mid \mathscr{F}_{T}\right]\right]=\frac{1}{\sigma} \mathbb{E}\left[\Phi\left(S_{T}\right)\left(\frac{W_{Y_{T}}^{2}}{Y_{T}}-1\right)\right],
$$

where the last equality holds by (3.4). Finally, for the arguments to remove the smoothness on $\Phi$, we need

$$
\mathbb{E}\left[\left|\Phi\left(S_{T}\right) W_{Y_{T}}^{2} / Y_{T}\right|\right]^{2} \leq \mathbb{E}\left[\left|\Phi\left(S_{T}\right)\right|^{2}\right] \mathbb{E}\left[W_{Y_{T}}^{4} / Y_{T}^{2}\right]=\mathbb{E}\left[\left|\Phi\left(S_{T}\right)\right|^{2}\right] \mathbb{E}\left[W_{1}^{4}\right]<+\infty,
$$

which concludes.

\subsection{Gamma}

Next, we consider the second-order sensitivity with respect to the initial value, that is, the delta of the delta. In the derivation, we literally take "delta" of the delta formula of Proposition 3.1 .

Proposition 3.4. Assume $\mathbb{E}\left[S_{T}^{2} W_{Y_{T}}^{2} / Y_{T}^{2}\right]<+\infty$. Let $\Phi: \mathbb{R} \rightarrow \mathbb{R}$ be a measurable function such that $\mathbb{E}\left[\Phi\left(S_{T}\right)^{2}\right]<+\infty$ is locally uniformly bounded in $S_{0}>0$. Assume further that $\mathbb{E}\left[Y_{T}^{-4}\right]<+\infty$. Then, we have

$$
\frac{\partial^{2}}{\partial S_{0}^{2}} \mathbb{E}\left[\Phi\left(S_{T}\right)\right]=\frac{1}{\sigma^{2} S_{0}^{2}} \mathbb{E}\left[\Phi\left(S_{T}\right) \frac{1}{Y_{T}}\left(\frac{W_{Y_{T}}^{2}}{Y_{T}}-\sigma W_{Y_{T}}-1\right)\right] .
$$


Proof. As before, it suffices to show the result with $\Phi \in C_{b}^{2}(\mathbb{R} ; \mathbb{R})$. In view of Proposition 3.1, we have

$$
\frac{\partial^{2}}{\partial S_{0}^{2}} \mathbb{E}\left[\Phi\left(S_{T}\right)\right]=\frac{\partial}{\partial S_{0}}\left(\frac{1}{\sigma S_{0}} \mathbb{E}\left[\Phi\left(S_{T}\right) \frac{W_{Y_{T}}}{Y_{T}}\right]\right) .
$$

Then, by a similar argument to the previous proofs, we have

$$
\begin{aligned}
\frac{\partial}{\partial S_{0}} \mathbb{E}\left[\Phi\left(S_{T}\right) \frac{W_{Y_{T}}}{Y_{T}}\right] & =\mathbb{E}\left[\frac{\partial}{\partial S_{0}} \Phi\left(S_{T}\right) \frac{W_{Y_{T}}}{Y_{T}}\right] \\
& =\frac{1}{S_{0}} \mathbb{E}\left[\Phi^{\prime}\left(S_{T}\right) \frac{S_{T} W_{Y_{T}}}{Y_{T}}\right] \\
& =\frac{1}{\sigma S_{0}} \mathbb{E}\left[\mathbb{E}\left[\Phi\left(S_{T}\right) \delta\left(W_{Y_{T}}\right) \mid \mathscr{F}_{T}\right] \frac{1}{Y_{T}^{2}}\right] \\
& =\frac{1}{\sigma S_{0}} \mathbb{E}\left[\Phi\left(S_{T}\right) \frac{W_{Y_{T}}^{2}-Y_{T}}{Y_{T}^{2}}\right]
\end{aligned}
$$

where the interchange of the derivative and the expectation can be justified by the dominated convergence theorem. Finally, for the arguments to remove the smoothness on $\Phi$, we need

$$
\mathbb{E}\left[\left|\Phi\left(S_{T}\right) W_{Y_{T}}^{4} / Y_{T}^{4}\right|\right]^{2} \leq \mathbb{E}\left[\Phi\left(S_{T}\right)^{2}\right] \mathbb{E}\left[Y_{T}^{-4}\right] \mathbb{E}\left[W_{1}^{8}\right]<+\infty
$$

which completes the proof.

\subsection{Delta of Asian}

Notice that by setting $Y_{t}=t$, our formulas recover the counterparts in the Black-Scholes framework. We may say, and indeed as our intuition, that in the derivation of the logarithmic derivatives, it does not matter how the clock is governed, but matters only where the Brownian motion is at the end.

A delta formula of the Asian options is derived below. Interestingly, even such a path dependent payoff yields a similar form to its counterpart in the Black-Scholes, which can be found in [22].

Proposition 3.5. Fix $T>0$, and define

$$
F_{1}:=\int_{0}^{T} S_{u} d u, \quad F_{2}:=\int_{0}^{T} S_{u} Y_{u} d u, \quad F_{3}:=\int_{0}^{T} S_{u} Y_{u}^{2} d u .
$$

Let $\Phi: \mathbb{R} \rightarrow \mathbb{R}$ be a measurable function such that $\mathbb{E}\left[\Phi\left(F_{1} / T\right)^{2}\right]$ is locally uniformly bounded in $S_{0}>0$. Assume further that $\left\{Y_{t}: t \in[0, T]\right\}$ is a non-decreasing Lévy process with Lévy measure $v$ defined on $\mathbb{R}_{+}$, and that there exist $p>1$ and $s>1$ with $q(s):=(1-1 / s)^{-1}$ such that

$$
\int_{z>1} e^{4 \xi z} v(d z)<+\infty
$$

where $\xi:=\left(4 p^{2} s^{2} \sigma^{2}+p s(0 \vee \theta)\right) \vee\left(2 p^{2} q(s)^{2} \sigma^{2}+p q(s)(0 \vee \theta)\right)$, and

$$
\mathbb{E}\left[Y_{\varepsilon}^{-4 p q(s)} e^{8 p^{2} q(s)^{2} \sigma^{2} Y_{\varepsilon}}\right]<+\infty
$$

for some $\varepsilon \in(0, T)$. Then, we have

$$
\frac{\partial}{\partial S_{0}} \mathbb{E}\left[\Phi\left(F_{1} / T\right)\right]=\frac{1}{S_{0}} \mathbb{E}\left[\Phi\left(F_{1} / T\right)\left(\frac{W_{Y_{T}} F_{1}}{\sigma F_{2}}+\frac{F_{1} F_{3}}{F_{2}^{2}}-1\right)\right] .
$$

Proof. As so far, we proceed with the proof in the case $\Phi \in C_{b}^{2}(\mathbb{R} ; \mathbb{R})$. First, observe that

$$
\frac{\partial}{\partial S_{0}} \mathbb{E}\left[\Phi\left(F_{1} / T\right)\right]=\mathbb{E}\left[\frac{\partial}{\partial S_{0}} \Phi\left(F_{1} / T\right)\right]=\frac{1}{S_{0}} \mathbb{E}\left[\Phi^{\prime}\left(F_{1} / T\right) F_{1} / T\right],
$$


where the interchange of the derivative and the expectation is justified by the dominated convergence theorem as before with $\mathbb{E}\left[F_{1}^{2}\right]<+\infty$. To prove this, observe that

$$
\begin{aligned}
\mathbb{E}\left[F_{1}^{2}\right] & \leq \mathbb{E}\left[\int_{0}^{T} S_{u}^{2} d u\right]=\int_{0}^{T} \mathbb{E}\left[S_{u}^{2}\right] d u \\
& \leq S_{0}^{2} e^{2 r T} \int_{0}^{T} \exp \left[u\left(\varphi_{Y_{1}}\left(-2 i\left(\theta-\sigma^{2}\right)\right)-\varphi_{Y_{1}}\left(-i\left(\theta+\sigma^{2} / 2\right)\right)\right)\right] d u<+\infty,
\end{aligned}
$$

where we have used the Cauchy-Schwarz inequality, the Fubini theorem, and the infinite divisibility of Lévy processes $\varphi_{Y_{t}}=t \varphi_{Y_{1}}$.

To continue our proof, given $\mathscr{F}_{T}$, note first that

$$
\int_{0}^{Y_{T}} D_{s} F_{1} d s=\sigma \int_{0}^{T} \int_{0}^{Y_{T}} S_{u} \mathbb{1}\left(s \leq Y_{u}\right) d s d u=\sigma F_{2}
$$

and

$$
\int_{0}^{Y_{T}} D_{s} F_{2} d s=\sigma \int_{0}^{T} \int_{0}^{Y_{T}} S_{u} Y_{u} \mathbb{1}\left(s \leq Y_{u}\right) d s d u=\sigma F_{3},
$$

where the interchanges of the Malliavin derivative and the Lebesgue integral hold true. (See, for example, Property P2 of [15].) By using the identity

$$
\delta\left(F_{1}\right)=W_{Y_{T}} F_{1}-\sigma F_{2},
$$

we get

$$
\delta\left(\frac{F_{1}}{F_{2}}\right)=\frac{\delta\left(F_{1}\right)}{F_{2}}+\frac{F_{1} \int_{0}^{Y_{T}} D_{s} F_{2} d s}{F_{2}^{2}}=\frac{W_{Y_{T}} F_{1}}{F_{2}}+\sigma \frac{F_{1} F_{3}}{F_{2}^{2}}-\sigma .
$$

The desired formula now follows from

$$
\mathbb{E}\left[\Phi^{\prime}\left(F_{1} / T\right) F_{1} / T \mid \mathscr{F}_{T}\right]=\mathbb{E}\left[\Phi\left(F_{1} / T\right) \delta\left(\frac{F_{1}}{\int_{0}^{Y_{T}} D_{s} F_{1} d s}\right) \mid \mathscr{F}_{T}\right],
$$

due to the conditional integration-by-parts formula (2.5) with $h_{t} \equiv 1$.

Concerning the integrability and the arguments to remove the smoothness of $\Phi$, by the Cauchy-Schwartz inequality with $\mathbb{E}\left[\Phi\left(F_{1} / T\right)^{2}\right]<+\infty$, it suffices to guarantee the existence of $\mathbb{E}\left[W_{Y_{T}}^{4}\left(F_{1} / F_{2}\right)^{4}\right]$ and $\mathbb{E}\left[\left(F_{1} F_{3} / F_{2}^{2}\right)^{4}\right]$. Since $F_{3} \leq Y_{T} F_{2}$, a.s., it further suffices to check the existence of $\mathbb{E}\left[W_{Y_{T}}^{4}\left(F_{1} / F_{2}\right)^{4}\right]$ and $\mathbb{E}\left[Y_{T}^{2}\left(F_{1} / F_{2}\right)^{4}\right]$. Let $q(p):=(1-1 / p)^{-1}$ so that $(p, q(p))$ are conjugate exponents. By the Hölder inequality, we have

$$
\mathbb{E}\left[W_{Y_{T}}^{4}\left(F_{1} / F_{2}\right)^{4}\right] \leq \mathbb{E}\left[\left|W_{Y_{T}}\right|^{4 q(p)}\right]^{1 / q(p)} \mathbb{E}\left[\left(F_{1} / F_{2}\right)^{4 p}\right]^{1 / p},
$$

and

$$
\mathbb{E}\left[Y_{T}^{4}\left(F_{1} / F_{2}\right)^{4}\right] \leq \mathbb{E}\left[Y_{T}^{4 q(p)}\right]^{1 / q(p)} \mathbb{E}\left[\left(F_{1} / F_{2}\right)^{4 p}\right]^{1 / p} .
$$

Hence, we investigate $\mathbb{E}\left[\left(F_{1} / F_{2}\right)^{4 p}\right]$. By the Hölder inequality, we have

$$
\mathbb{E}\left[\left(F_{1} / F_{2}\right)^{4 p}\right] \leq \mathbb{E}\left[F_{1}^{4 p s}\right]^{1 / s} \mathbb{E}\left[F_{2}^{-4 p q(s)}\right]^{1 / q(s)} .
$$

Recalling the formula

$$
\mathbb{P}\left[\sup _{t \in[0, T]} W_{t} \in B\right]=\int_{0}^{+\infty} \mathbb{1}_{B}(x) \frac{2}{\sqrt{2 \pi T}} \exp \left[-\frac{x^{2}}{2 T}\right] d x, \quad B \in \mathscr{B}\left(\mathbb{R}_{+}\right),
$$

where $\mathscr{B}\left(\mathbb{R}_{+}\right)$is the Borel $\sigma$-field of $\mathbb{R}_{+}$, we get

$$
\begin{aligned}
\mathbb{E}\left[F_{1}^{4 p s}\right] & \leq T^{4 p s} e^{4 p s r T} \mathbb{E}\left[e^{4 p s \sup _{t \in[0, T]}\left(\theta Y_{t}+\sigma W_{Y_{t}}+\varphi_{Y_{t}}\left(-i\left(\theta+\sigma^{2} / 2\right)\right)\right)}\right] \\
& \leq T^{4 p s} e^{4 p s r T} e^{4 p s \sup _{t \in[0, T]} \varphi_{Y_{t}}\left(-i\left(\theta+\sigma^{2} / 2\right)\right)} \mathbb{E}\left[e ^ { 4 p s \operatorname { s u p } _ { t \in [ 0 , T ] } ( \theta Y _ { t } ) } \mathbb { E } \left[e^{\left.\left.4 p s \sigma \sup _{t \in[0, T]} W_{Y_{t}} \mid \mathscr{F}_{T}\right]\right]}\right.\right. \\
& \leq 2 T^{4 p s} e^{4 p s r T} e^{4 p s \sup _{t \in[0, T]} \varphi_{Y_{t}}\left(-i\left(\theta+\sigma^{2} / 2\right)\right)} \mathbb{E}\left[e^{4 p s \sup _{t \in[0, T]}\left(\theta Y_{t}\right)+16 p^{2} s^{2} \sigma^{2} Y_{T}}\right] \\
& \leq 2 T^{4 p s} e^{4 p s r T} e^{4 p s \sup _{t \in[0, T]} \varphi_{Y_{t}}\left(-i\left(\theta+\sigma^{2} / 2\right)\right)} \mathbb{E}\left[e^{\left(16 p^{2} s^{2} \sigma^{2}+4 p s(0 \vee \theta)\right) Y_{T}}\right] .
\end{aligned}
$$


Hence, we get that $\mathbb{E}\left[F_{1}^{4 p s}\right]<+\infty$ if $\mathbb{E}\left[e^{\left(16 p^{2} s^{2} \sigma^{2}+4 p s(0 \vee \theta)\right) Y_{T}}\right]<+\infty$. Next, we investigate $\mathbb{E}\left[F_{2}^{-4 p q(s)}\right]$. For ease in notation, write $\tau:=-4 p q(s)$. Pick an arbitrary $\varepsilon \in(0, T)$. First, since $\mathbb{P}\left(S_{t}>0\right)=\mathbb{P}\left(Y_{t}>0\right)=1$ for each $t \in(0, T]$, we have $\mathbb{E}\left[F_{2}^{\tau}\right] \leq(T-\varepsilon)^{\tau} \mathbb{E}\left[Y_{\varepsilon}^{\tau}\left(\inf _{t \in[\varepsilon, T]} S_{t}\right)^{\tau}\right]$, and

$$
\mathbb{E}\left[Y_{\mathcal{E}}^{\tau}\left(\inf _{t \in[\varepsilon, T]} S_{t}\right)^{\tau}\right] \leq \mathbb{E}\left[Y_{\mathcal{E}}^{\tau} e^{\tau \inf _{t \in[\varepsilon, T]}\left(\theta Y_{t}+\varphi_{Y_{t}}\left(-i\left(\theta+\sigma^{2} / 2\right)\right)\right.} \mathbb{E}\left[e^{\tau \inf _{t \in\left[Y_{\varepsilon}, Y_{T}\right]} \sigma W_{t}} \mid \mathscr{F}_{T}\right]\right]
$$

Then, by using the independence of increments and formula (3.6), we get

$$
\mathbb{E}\left[e^{\tau \inf _{t \in\left[Y_{\varepsilon}, Y_{T}\right]}\left(\sigma W_{t}\right)} \mid \mathscr{F}_{T}\right]=e^{\frac{1}{2} \tau^{2} \sigma^{2} Y_{\varepsilon}} \mathbb{E}\left[e^{-\tau \sigma \sup _{t \in\left[Y_{\varepsilon}, Y_{T}\right]}\left(W_{t}-W_{Y_{\varepsilon}}\right)} \mid \mathscr{F}_{T}\right] \leq 2 e^{\frac{1}{2} \tau^{2} \sigma^{2} Y_{T}},
$$

where the inequality holds by the fact $\mathbb{P}\left(Y_{T}-Y_{\varepsilon}>0\right)=1$. Hence, we get

$$
\begin{aligned}
\mathbb{E}\left[Y_{\varepsilon}^{\tau}\left(\inf _{t \in[\varepsilon, T]} S_{t}\right)^{\tau}\right] \leq & 2 \mathbb{E}\left[Y_{\varepsilon}^{\tau} \exp \left[\tau \inf _{t \in[\varepsilon, T]}\left(\theta Y_{t}+\varphi_{Y_{t}}\left(-i\left(\theta+\sigma^{2} / 2\right)\right)\right)+Y_{T} \tau^{2} \frac{\sigma^{2}}{2}\right]\right] \\
\leq & 2 e^{\tau \inf _{t \in[\varepsilon, T]} \varphi_{Y_{t}}\left(-i\left(\theta+\sigma^{2} / 2\right)\right)} \\
& \times \mathbb{E}\left[Y_{\varepsilon}^{\tau} \exp \left[\tau \inf _{t \in[\varepsilon, T]}\left(\theta\left(Y_{t}-Y_{\varepsilon}\right)\right)+\left(\tau^{2} \sigma^{2} / 2+\tau \theta\right) Y_{\varepsilon}+\left(Y_{T}-Y_{\varepsilon}\right) \tau^{2} \frac{\sigma^{2}}{2}\right]\right] \\
= & 2 e^{\tau \inf _{t \in[\varepsilon, T]} \varphi_{Y_{t}}\left(-i\left(\theta+\sigma^{2} / 2\right)\right)} \mathbb{E}\left[Y_{\varepsilon}^{\tau} e^{\left(\tau^{2} \sigma^{2} / 2+\tau \theta\right) Y_{\varepsilon}}\right] \mathbb{E}\left[e^{\left(\tau^{2} \sigma^{2} / 2-(0 \wedge-\tau \theta)\right) Y_{T-\varepsilon}}\right],
\end{aligned}
$$

where the equality holds by the independence and the stationarity of increments of $\left\{Y_{t}: t \in[0, T]\right\}$. Hence, the expectation $\mathbb{E}\left[F_{2}^{\tau}\right]$ is well defined if there exists $\varepsilon \in(0, T)$ such that $\mathbb{E}\left[Y_{\varepsilon}^{\tau} e^{\tau^{2} \sigma^{2} / 2 Y_{\varepsilon}}\right]<+\infty$ and if $\mathbb{E}\left[e^{\left(\tau^{2} \sigma^{2} / 2-(0 \wedge-\tau \theta)\right) Y_{T}}\right]<+\infty$. Theorem 25.3 of Sato [25] concludes the proof.

\subsection{Greeks for Baskets}

In this subsection, we consider a basket of correlated asset prices, and its sensitivity with respect to the correlation parameter $\rho$, and its so-called cross-gamma, that is, $\left(\partial^{2} /\left(\partial S_{0}^{(1)} \partial S_{0}^{(2)}\right)\right)$. We here restrict ourselves to a basket of only two assets, while our results can easily be extended to a higher dimension.

Fix $\rho \in(-1,1)$ and set $\eta(\rho):=\sqrt{1-\rho^{2}}$. Define

$$
\left[\begin{array}{l}
X_{t}^{(1)} \\
X_{t}^{(2)}
\end{array}\right]:=\left[\begin{array}{l}
\theta_{1} Y_{t}+\sigma_{1} W_{Y_{t}}^{(1)}-\varphi_{Y_{t}}\left(-i\left(\theta_{1}+\sigma_{1}^{2} / 2\right)\right) \\
\theta_{2} Y_{t}+\sigma_{2}\left(\rho W_{Y_{t}}^{(1)}+\eta(\rho) W_{Y_{t}}^{(2)}\right)-\varphi_{Y_{t}}\left(-i\left(\theta_{2}+\sigma_{2}^{2} / 2\right)\right)
\end{array}\right]
$$

and

$$
\left[F_{1}, F_{2}\right]^{\prime}:=\left[S_{0}^{(1)} \exp \left(X_{T}^{(1)}\right), S_{0}^{(2)} \exp \left(X_{T}^{(2)}\right)\right]^{\prime},
$$

where $S_{0}^{(1)}$ and $S_{0}^{(2)}$ are positive constants. Set $F:=a_{1} F_{1}+a_{2} F_{2}$, for some real $a_{1}$ and $a_{2}$. Then, we have the following.

Proposition 3.6. Let $\Phi: \mathbb{R} \mapsto \mathbb{R}$ be a measurable function such that $\mathbb{E}\left[\Phi(F)^{2}\right]$ is locally uniformly bounded in $S_{0}^{(1)}>0$ and $S_{0}^{(2)}>0$. Then, it holds that

$$
\frac{\partial}{\partial \rho} \mathbb{E}[\Phi(F)]=\frac{1}{\eta(\rho)} \mathbb{E}\left[\Phi(F) \frac{W_{Y_{T}}^{(1)} W_{Y_{T}}^{(2)}-\frac{\rho}{\eta(\rho)}\left(\left(W_{Y_{T}}^{(2)}\right)^{2}-Y_{T}\right)}{Y_{T}}\right] .
$$

If $\mathbb{E}\left[Y_{T}^{-2}\right]<+\infty$, then it holds that

$$
\frac{\partial^{2}}{\partial S_{0}^{(1)} \partial S_{0}^{(2)}} \mathbb{E}[\Phi(F)]=\frac{1}{\eta(\rho) \sigma_{1} \sigma_{2} S_{0}^{(1)} S_{0}^{(2)}} \mathbb{E}\left[\Phi(F) \frac{1}{Y_{T}^{2}}\left(W_{Y_{T}}^{(1)} W_{Y_{T}}^{(2)}-\frac{\rho}{\eta(\rho)}\left(\left(W_{Y_{T}}^{(2)}\right)^{2}-Y_{T}\right)\right)\right] .
$$


Proof. In a similar manner to the previous proofs, it suffices to assume $\Phi \in C_{b}^{2}(\mathbb{R} ; \mathbb{R})$.

For the sensitivity with respect to the correlation parameter, we proceed

$$
\begin{aligned}
\frac{\partial}{\partial \rho} \mathbb{E}[\Phi(F)] & =a_{2} \sigma_{2} \mathbb{E}\left[\Phi^{\prime}(F) F_{2}\left(W_{Y_{T}}^{(1)}-\frac{\rho}{\eta(\rho)} W_{Y_{T}}^{(2)}\right)\right] \\
& =\frac{1}{\eta(\rho)} \mathbb{E}\left[\int_{0}^{Y_{T}} D_{s}^{(2)} \Phi(F) \frac{W_{Y_{T}}^{(1)}-\frac{\rho}{\eta(\rho)} W_{Y_{T}}^{(2)}}{Y_{T}} d s\right] \\
& =\frac{1}{\eta(\rho)} \mathbb{E}\left[\frac{1}{Y_{T}} \mathbb{E}\left[\Phi(F) \delta_{2}\left(W_{Y_{T}}^{(1)}-\frac{\rho}{\eta(\rho)} W_{Y_{T}}^{(2)}\right) \mid \mathscr{F}_{T}\right]\right] \\
& =\frac{1}{\eta(\rho)} \mathbb{E}\left[\frac{1}{Y_{T}} \mathbb{E}\left[\Phi(F)\left(W_{Y_{T}}^{(1)} W_{Y_{T}}^{(2)}-\frac{\rho}{\eta(\rho)}\left(\left(W_{Y_{T}}^{(2)}\right)^{2}-Y_{T}\right)\right) \mid \mathscr{F}_{T}\right]\right],
\end{aligned}
$$

where the interchange of the derivative and the expectation at the first equality can be justified by standard uniform integrability arguments, where the second equality can be justified by $D_{s}^{(2)} \Phi(F)=\Phi^{\prime}(F) a_{2} F_{2} \sigma_{2} \eta(\rho)$, and where the third equality holds by the conditional integration-by-parts formula (2.5) with $h_{t} \equiv 1$.

For the cross-gamma, we start with deriving an expression for $\left(\partial / \partial S_{0}^{(2)}\right) \mathbb{E}[\Phi(F)]$, which can be done by

$$
\begin{aligned}
\frac{\partial}{\partial S_{0}^{(2)}} \mathbb{E}[\Phi(F)] & =\frac{a_{2}}{S_{0}^{(2)}} \mathbb{E}\left[\Phi^{\prime}(F) F_{2}\right] \\
& =\frac{1}{S_{0}^{(2)} \eta(\rho) \sigma_{2}} \mathbb{E}\left[\frac{1}{Y_{T}} \mathbb{E}\left[\int_{0}^{Y_{T}} D_{s}^{(2)} \Phi(F) d s \mid \mathscr{F}_{T}\right]\right] \\
& =\frac{1}{S_{0}^{(2)} \eta(\rho) \sigma_{2}} \mathbb{E}\left[\Phi(F) \frac{W_{Y_{T}}^{(2)}}{Y_{T}}\right],
\end{aligned}
$$

where the last equality holds by $D_{s}^{(2)} \Phi(F)=\Phi^{\prime}(F) a_{2} F_{2} \sigma_{2} \eta(\rho)$. With the identity $D_{s}^{(1)} \Phi(F)=\Phi^{\prime}(F)\left(a_{1} \sigma_{1} F_{1}+\right.$ $a_{2} \sigma_{2} \rho F_{2}$ ), we have

$$
\Phi^{\prime}(F) a_{1} \sigma_{1} F_{1}=\frac{1}{Y_{T}}\left(\int_{0}^{Y_{T}} D_{s}^{(1)} \Phi(F) d s-\frac{\rho}{\eta(\rho)} \int_{0}^{Y_{T}} D_{s}^{(2)} \Phi(F) d s\right) .
$$

Using this identity, we proceed

$$
\begin{aligned}
\frac{\partial^{2}}{\partial S_{0}^{(1)} \partial S_{0}^{(2)}} \mathbb{E}[\Phi(F)] & =\frac{a_{1}}{S_{0}^{(1)} S_{0}^{(2)} \eta(\rho) \sigma_{2}} \mathbb{E}\left[\Phi^{\prime}(F) F_{1} \frac{W_{Y_{T}}^{(2)}}{Y_{T}}\right] \\
& =\frac{1}{S_{0}^{(1)} S_{0}^{(2)} \eta(\rho) \sigma_{1} \sigma_{2}} \mathbb{E}\left[\frac{1}{Y_{T}^{2}} \int_{0}^{Y_{T}}\left(D_{s}^{(1)} \Phi(F)-\frac{\rho}{\eta(\rho)} D_{s}^{(2)} \Phi(F)\right) W_{Y_{T}}^{(2)} d s\right],
\end{aligned}
$$

which yields the desired equality by the conditional integration-by-parts formula (2.5) with $h_{t} \equiv 1$. The proof is complete. (In our setting, regardless of the order of the partial derivatives and the choice of the Skorohod integrals, we arrive at the same formula.)

Remark 3.7. For all the formulas of this section, we have imposed the assumptions on the $L^{2}(\Omega)$-integrability of the payoff and on the finite negative moment $\mathbb{E}\left[Y_{T}^{-p}\right]<+\infty$, for some $p>0$. As a matter of course, with a higher order integrability of the payoff and with the use of the Hölder inequality (instead of the Cauchy-Schwarz inequality) just as in the proof of Proposition 3.5, we may decrease the order $p$ of the negative moment to a certain extent. In particular, in the case of uniformly bounded payoffs, such as digital options (to be tested in our numerical experiments below), the order $p$ can be taken arbitrarily small. Moreover, the order $p$ has been increased in order to remove the smoothness of the payoff function $\Phi$. Hence, we can again decrease the order $p$ when dealing with smooth $\Phi$ s. For the reader's convenience, let us here investigate the negative moments for the three candidates given in Example 2.1, 2.2, and 2.3 For the gamma process, by using the density function $(2.2)$, we can easily derive that $\mathbb{E}\left[Y_{T}^{-p}\right]<+\infty$ if and only if $p \in(0, a T)$. Meanwhile, it is well known that the inverse Gaussian distribution has the density

$$
f_{Y_{t}}(x)=\frac{\delta t e^{\delta \gamma t}}{\sqrt{2 \pi} x^{3 / 2}} \exp \left[-\frac{1}{2}\left(\delta^{2} t^{2} x^{-1}+\gamma^{2} x\right)\right], \quad x \in(0,+\infty),
$$


where parameters are the same as given in Example 2.2. Hence, $\mathbb{E}\left[Y_{T}^{-p}\right]<+\infty$ for every $p \in(0,+\infty)$. Finally, for the integrated CIR square root process, we use the identity

$$
\mathbb{E}\left[Y_{T}^{-p}\right]=\frac{1}{\Gamma(p)} \int_{0}^{+\infty} s^{p-1} \mathbb{E}\left[e^{-s Y_{T}}\right] d s=\frac{1}{\Gamma(p)} \int_{0}^{+\infty} s^{p-1} e^{A(T, i s) z_{0}} B(T, i s) d s
$$

where the functions are as given in Example 2.3. We can show that $A(T, i s) \sim-2 \sqrt{2 s} / \lambda, B(T, i s) \sim e^{-\sqrt{2 s} \kappa \eta T / \lambda}$, and thus $\mathbb{E}\left[e^{-s Y_{T}}\right] \sim e^{-\sqrt{2 s}\left(2 z_{0}+\kappa \eta T\right) / \lambda}$ as $s \uparrow+\infty$. This implies that the negative moment is well defined for every order. Interestingly, the above observations indicate that the inverse Gaussian process and the integrated CIR square root process cannot stay very low for long.

\subsection{Numerical Illustration}

We test our formulas on a digital payoff $\Phi(x)=\mathbb{1}(x>K)$ with the normal inverse Gaussian model described in Example 2.2. In the numerical results given below, "FD" indicates the Monte Carlo convergence of the finite difference counterparts. For the delta, the vega, the gamma, and the Asian delta, we set model parameters $S_{0}=100, K=1.1 S_{0}$, $\alpha=8, \beta=-3$, and $\delta=0.2$. For the baskets, we use the same inverse Gaussian process and set $S_{0}^{(1)}=S_{0}^{(2)}=100$, $\rho=0.3, a_{1}=a_{2}=1$, and $K=2.2 S_{0}^{(1)}$. We fix $T=1$ and $r=0.05$ throughout. The figures and the variance ratios evidently indicate a faster convergence of our Greeks formulae, compared to the finite difference approximations. Note that the finite difference approximations are in general biased.

\section{Multidimensional Density Estimation}

This section treats the problem of obtaining a Monte Carlo estimation of the value of the density for a multidimensional time-changed Brownian motion model. Let us begin with some additional notations on the Malliavin calculus. Fix $T>0, d_{1} \in \mathbb{N}$, and $d_{2} \in \mathbb{N}$. Let $\left\{W_{t}: t \geq 0\right\}$ be a standard Brownian motion in $\mathbb{R}^{d_{1}}$ and define by $F:=\left(F_{1}, \ldots, F_{d_{2}}\right)^{\prime}$ a random vector in $\mathbb{R}^{d_{2}}$, measurable with respect to $\mathscr{G}_{T}$. We denote by $\gamma_{F}$ the Malliavin matrix of $F$ given $\mathscr{F}_{T}$, whose $\left(k_{1}, k_{2}\right)$-entry is

$$
\left(\gamma_{F}\right)_{k_{1}, k_{2}}=\int_{0}^{Y_{T}}\left\langle D_{s} F_{k_{1}}, D_{s} F_{k_{2}}\right\rangle d s
$$

Define for $k=1, \ldots, d_{2}$,

$$
H_{(k)}(F):=\sum_{l=1}^{d_{1}} \sum_{m=1}^{d_{2}} \delta_{l}\left(\left(\gamma_{F}^{-1}\right)_{k, m} D^{(l)} F_{m}\right)
$$

and set

$$
H(F):=\left[H_{(1)}(F), \ldots, H_{\left(d_{2}\right)}(F)\right]^{\prime} .
$$

We denote by $a_{d}$ the area of the unit sphere in $\mathbb{R}^{d}$, and define $c_{2}:=a_{2}$ and $c_{d}:=(d-2) a_{d}$ for $d \geq 3$. Moreover, for each $d \geq 2$, define a map $Q_{d}: \mathbb{R}^{d} \mapsto \mathbb{R}$ by $Q_{2}(x):=a_{2} \ln \|x\|$, and for $d \geq 3, Q_{d}(x):=-a_{d}\|x\|^{2-d}$. Then, we get for $k=1, \ldots, d$,

$$
\nabla Q_{d}(x)=c_{d} \frac{x}{\|x\|^{d}} .
$$

The following is a key tool due to Malliavin and Thalmaier (our formulation follows from Bally and Caramellino [2]). We illustrate, in Example 4.2, how the algebra actually proceeds with numerical results.

Theorem 4.1. (Kohatsu-Higa and Yasuda [19]) Assume that there exists $p>1$ such that for every $c>0$,

$$
\sup _{\|x\| \leq c} \mathbb{E}\left[\left\|\nabla Q_{d_{2}}(F-x)\right\|^{\frac{p}{p-1}}+\left|Q_{d_{2}}(F-x)\right|^{\frac{p}{p-1}}\right]<+\infty .
$$

Then, the density $p_{F}(\cdot)$ of $F$ is given by

$$
p_{F}(x)=\mathbb{E}\left[\left\langle\nabla Q_{d_{2}}(F-x), H(F)\right\rangle\right] .
$$


The idea of the above result is to use the fact that in the sense of generalized functions, one has that (see, for example, Evans [14])

$$
\nabla Q_{d_{2}}(y-x)=\delta_{x}(y)
$$

Therefore, the above result can be understood as an application of the integration-by-parts formula.

Example 4.2. Consider the two-dimensional model investigated in Section 3.5. Set

$$
F:=\left[F_{1}, F_{2}\right]^{\prime}:=\left[S_{0}^{(1)} \exp \left(X_{T}^{(1)}\right), S_{0}^{(2)} \exp \left(X_{T}^{(2)}\right)\right]^{\prime},
$$

where $S_{0}^{(1)}$ and $S_{0}^{(2)}$ are positive constants, as before. Then, given $\mathscr{F}_{T}$, we have for $s \in\left[0, Y_{T}\right]$,

$$
D_{s} F_{k}= \begin{cases}\sigma_{1} F_{1}[1,0]^{\prime}, & \text { if } k=1, \\ \sigma_{2} F_{2}[\rho, \eta(\rho)]^{\prime}, & \text { if } k=2,\end{cases}
$$

and

$$
D_{s} F_{k}^{-1}=-F_{k}^{-2} D_{s} F_{k}= \begin{cases}-\sigma_{1} F_{1}^{-1}[1,0]^{\prime}, & \text { if } k=1, \\ -\sigma_{2} F_{2}^{-1}[\rho, \eta(\rho)]^{\prime}, & \text { if } k=2 .\end{cases}
$$

Hence, the Malliavin matrix $\gamma_{F}$ of the random vector $F$ given $\mathscr{F}_{T}$ is

$$
\gamma_{F}=\int_{0}^{Y_{T}}\left[\begin{array}{cc}
\left\|D_{s} F_{1}\right\|^{2} & \left\langle D_{s} F_{1}, D_{s} F_{2}\right\rangle \\
\left\langle D_{s} F_{1}, D_{s} F_{2}\right\rangle & \left\|D_{s} F_{2}\right\|^{2}
\end{array}\right] d s=Y_{T}\left[\begin{array}{cc}
\sigma_{1}^{2} F_{1}^{2} & \sigma_{1} \sigma_{2} F_{1} F_{2} \rho \\
\sigma_{1} \sigma_{2} F_{1} F_{2} \rho & \sigma_{2}^{2} F_{2}^{2}
\end{array}\right],
$$

while its inverse almost surely exists and is given by

$$
\gamma_{F}^{-1}=\frac{1}{Y_{T} \sigma_{1}^{2} \sigma_{2}^{2} F_{1}^{2} F_{2}^{2} \eta(\rho)^{2}}\left[\begin{array}{cc}
\sigma_{2}^{2} F_{2}^{2} & -\sigma_{1} \sigma_{2} F_{1} F_{2} \rho \\
-\sigma_{1} \sigma_{2} F_{1} F_{2} \rho & \sigma_{1}^{2} F_{1}^{2}
\end{array}\right] .
$$

Given $\mathscr{F}_{T}$, using the conditional integration-by-parts formula (2.5) with $h_{t} \equiv 1$, we get

$$
\delta_{l}\left(F_{m}^{-1}\right)=F_{m}^{-1} \delta_{l}(1)-\int_{0}^{Y_{T}} D_{s}^{(l)} F_{m}^{-1} d s= \begin{cases}F_{1}^{-1}\left(W_{Y_{T}}^{(1)}-\sigma_{1} Y_{T}\right), & \text { if }(l, m)=(1,1), \\ F_{2}^{-1}\left(W_{Y_{T}}^{(1)}-\sigma_{2} \rho Y_{T}\right), & \text { if }(l, m)=(1,2), \\ F_{1}^{-1} W_{Y_{T}}^{(2)}, & \text { if }(l, m)=(2,1), \\ F_{2}^{-1}\left(W_{Y_{T}}^{(2)}-\sigma_{2} \eta(\rho) Y_{T}\right), & \text { if }(l, m)=(2,2) .\end{cases}
$$

Hence, we get

$$
\begin{aligned}
H_{(1)}(F)= & \delta_{1}\left(\left(\gamma_{F}^{-1}\right)_{1,1} D^{(1)} F_{1}\right)+\delta_{1}\left(\left(\gamma_{F}^{-1}\right)_{1,2} D^{(1)} F_{2}\right) \\
& +\delta_{2}\left(\left(\gamma_{F}^{-1}\right)_{1,1} D^{(2)} F_{1}\right)+\delta_{2}\left(\left(\gamma_{F}^{-1}\right)_{1,2} D^{(2)} F_{2}\right) \\
= & \frac{\delta_{1}\left(F_{1}^{-1}\right)}{\sigma_{1} Y_{T} \eta(\rho)^{2}}-\frac{\rho^{2} \delta_{1}\left(F_{1}^{-1}\right)}{\sigma_{1} Y_{T} \eta(\rho)^{2}}+0-\frac{\rho \delta_{2}\left(F_{1}^{-1}\right)}{\sigma_{1} Y_{T} \eta(\rho)} \\
= & \frac{W_{Y_{T}}^{(1)}-\frac{\rho}{\eta(\rho)} W_{Y_{T}}^{(2)}-\sigma_{1} Y_{T}}{F_{1} \sigma_{1} Y_{T}},
\end{aligned}
$$

and

$$
\begin{aligned}
H_{(2)}(F)= & \delta_{1}\left(\left(\gamma_{F}^{-1}\right)_{2,1} D^{(1)} F_{1}\right)+\delta_{1}\left(\left(\gamma_{F}^{-1}\right)_{2,2} D^{(1)} F_{2}\right) \\
& +\delta_{2}\left(\left(\gamma_{F}^{-1}\right)_{2,1} D^{(2)} F_{1}\right)+\delta_{2}\left(\left(\gamma_{F}^{-1}\right)_{2,2} D^{(2)} F_{2}\right) \\
= & -\frac{\rho \delta_{1}\left(F_{2}^{-1}\right)}{\sigma_{2} Y_{T} \eta(\rho)^{2}}+\frac{\rho \delta_{1}\left(F_{2}^{-1}\right)}{\sigma_{2} Y_{T} \eta(\rho)^{2}}+0+\frac{\delta_{2}\left(F_{2}^{-1}\right)}{\sigma_{2} Y_{T} \eta(\rho)} \\
= & \frac{W_{Y_{T}}^{(2)}-\sigma_{2} Y_{T} \eta(\rho)}{F_{2} \sigma_{2} Y_{T} \eta(\rho)} .
\end{aligned}
$$


Hence, we get for $x:=\left(x_{1}, x_{2}\right)^{\prime} \in \mathbb{R}^{2}$,

$$
\begin{aligned}
p_{F}(x) & =c_{2} \mathbb{E}\left[\mathbb{E}\left[\left\langle\frac{F-x}{\|F-x\|^{2}}, H(F)\right\rangle \mid \mathscr{F}_{T}\right]\right] \\
& =c_{2} \mathbb{E}\left[\frac{F_{1}-x_{1}}{\|F-x\|^{2}} \frac{W_{Y_{T}}^{(1)}-\frac{\rho}{\eta(\rho)} W_{Y_{T}}^{(2)}-\sigma_{1} Y_{T}}{F_{1} \sigma_{1} Y_{T}}+\frac{F_{2}-x_{2}}{\|F-x\|^{2}} \frac{W_{Y_{T}}^{(2)}-\sigma_{2} Y_{T} \eta(\rho)}{F_{2} \sigma_{2} Y_{T} \eta(\rho)}\right] .
\end{aligned}
$$

We can show that the above formula is well defined when there exists $p>1$ and $q>0$ such that

$$
\mathbb{E}\left[Y_{T}^{-p} e^{2 p\left(\left(-\theta_{1}+q\right) \vee\left(-\theta_{2}+p \sigma_{2}^{2}\right)\right) Y_{T}}\right]<+\infty .
$$

Notice that as in the case of the Greeks formulas of European type, the Markov feature of the time control process is not required in the derivation of the above estimator and we need only the sample of the marginal $Y_{T}$, but not the entire sample paths $\left\{Y_{t}: t \in[0, T]\right\}$. We present in Figure 2 and Figure 3 simulation results, respectively, of the variance gamma model and the normal inverse Gaussian model.

For two reasons, the above density estimation formulae outperforms the standard kernel density estimation, for example,

$$
\widehat{p}_{h}(x):=\frac{1}{h^{d_{1}}} \mathbb{E}\left[K\left(\frac{x-S_{T}}{h}\right)\right], \quad x \in \mathbb{R}^{d_{1}},
$$

where $K$ is a suitable kernel and where $h$ is the bandwidth. First, the kernel density estimation is only asymptotically unbiased as $h \downarrow 0$, while in reality $h$ can never be taken zero. On the other hand, our density estimators are intrinsically unbiased. Second, in the kernel density estimation, Monte Carlo summands provide almost no contribution to the convergence of Monte Carlo simulation unless realizations are very close to $x$. This problem turns out to be very serious when we set the bandwidth $h$ to be extremely small. In our formula, meanwhile, all the Monte Carlo realizations make an equal contribution.

A drawback of our formula is its infinite variance, due to the singularity of the denominator $\|F-x\|^{2}$. To get around this issue, we may employ an approximation technique studied in Kohatsu-Higa and Yasuda [19] also in our framework, in exchange for the lost of the unbiasedness. In such a case, the method is similar to the kernel density estimation but the rates of bias and variance and smaller than in the classical method due to the use of the conditional integration-by-parts formula.

\section{Concluding Remarks}

In this paper, we have computed formulas for the Greeks and the multidimensional density estimation for an asset price dynamics model defined with time-changed Brownian motions. Our approach is based on an application of Malliavin duality formulas on the Gaussian space conditioning on the jump component. We have noticed that the Markov property of the time-changing process is not required and that our formulas may recover the counterparts with the degenerate time clock.

On the sensitivity analysis for asset price models related to jump processes, a variety of different approaches have been proposed in the literature. In some cases, we may derive different formulas for a single object. For example, the variance gamma model can also be in the framework of [17], where different formulas are obtained for delta, gamma, and vega. It would be interesting to somehow investigate how those distinct formulas should be compared, for example, in terms of the Monte Carlo variance.

\section{Acknowledgements}

The authors would like to thank Japan Society for the Promotion of Science for Grant-in-Aid for Scientific Research 20740059 and 21340024. This work was largely carried out while RK was based at Center for the Study of Finance and Insurance, Osaka University. 


\section{References}

[1] Bally, V., Bavouzet, M. P., Messaoud, M. (2007) Integration by parts formula for locally smooth laws and applications to sensitivity computations, Annals of Applied Probability, 17(1) 33-66.

[2] Bally, V., Caramellino, L. (2006) Lower bounds for the density of Ito processes under weak regularity conditions, working paper.

[3] Barndorff-Nielsen, O.E. (1998) Processes of normal inverse Gaussian type, Finance and Stochastics, 2(1) 4168.

[4] Bichteler, K., Gravereaux, J. B., Jacod, J. (1987) Malliavin calculus for processes with jumps, Gordon and Breach Science Publishers, New York.

[5] Bismut, J. M. (1981) Martingales, the Malliavin calculus and hypoellipticity under general Hörmander's conditions, Z. Wahrsch. Verw. Gebiete, 56(4) 469-505.

[6] Bismut, J. M. (1984) Large deviations and the Malliavin calculus, Birkhäuser, Boston.

[7] Carlen, E., Pardoux, E. (1990) Differential calculus and integration by parts on Poisson space. In: Albeverio, S., Blanchard, Ph., Testard, D. (eds.) Stochastics, algebra and analysis in classical and quantum dynamics (Marseille, 1988) (Math. Appl., vol. 59). Dordrecht: Kluwer Acad. Publ., 63-73.

[8] Carr, P., Geman, H., Madan, D., Yor, M. (2003) Stochastic Volatility for Lévy processes, Mathematical Finance, 13(3) 345-382.

[9] Carr, P., Madan, D. (1999) Option pricing and the fast Fourier transform, Journal of Computational Finance, 2(4) 61-73.

[10] Cass, T. R., Friz, P. K. (2007) The Bismut-Elworthy-Li formula for jump-diffusions and applications to Monte Carlo methods in finance, available at arXiv:math/0604311v3.

[11] Cox, J.C., Ingersoll, J.E., Ross, S.A. (1985) A theory of the term structure of interest rates, Econometrica, 53(2) 385-407.

[12] Davis, M.H.A., Johansson, M.P. (2006) Malliavin Monte Carlo Greeks for jump diffusions, Stochastic Processes and their Applications, 116(1) 101-129.

[13] El-Khatib, Y., Privault, N. (2004) Computations of Greeks in a market with jumps via the Malliavin calculus, Finance and Stochastics, 8(2) 161-179.

[14] L. C. Evans (1998) Partial Differential Equations. Graduate Studies in Mathematics. Volume 19. American Mathematical Society, 1998.

[15] Fournié, E., Lasry, J. M., Lebuchoux, J., Lions, P. L., Touzi, N. (1999) Applications of Malliavin calculus to Monte Carlo methods in finance, Finance and Stochastics, 3(4) 391-412.

[16] Fu, M.C. (2007) Variance-Gamma and Monte Carlo, In: Advances in Mathematical Finance (eds. Fu, Jarrow, Yen, Elliott), 21-35, Birkhäuser.

[17] Kawai, R., Takeuchi, A., Greeks formulae for an asset price model with gamma processes, Mathematical Finance, to appear.

[18] Kawai, R., Takeuchi, A. (2010) Sensitivity analysis for averaged asset price dynamics with gamma processes, Statistics and Probability Letters, 80(1) 42-49.

[19] Kohatsu-Higa, A., Yasuda, K. (2009) Estimating multidimensional density functions using the MalliavinThalmaier formula, SIAM Journal on Numerical Analysis, 47(2) 1546-1575.

[20] Madan, D., Carr, P., Chang, E. (1998) The variance gamma process and option pricing, European Finance Review, 2, 79-105. 
[21] Malliavin, A., Thalmaier, A. (2006) Stochastic Calculus of Variations in Mathematical Finance, SpringerVerlag, Berlin.

[22] Montero, M., Kohatsu-Higa, A. (2003) Malliavin calculus applied to finance, Physica A, 320, 548-570.

[23] Nualart, D. (2006) The Malliavin Calculus and Related Topics, Springer-Verlag, Berlin.

[24] Rosiński, J. (1991) On a class of infinitely divisible processes represented as mixtures of Gaussian processes, In: Stable Processes and Related Topics, Progress in Probability, 25, Birkhäuser, Basel, 27-41.

[25] Sato, K. (1999) Lévy processes and infinitely divisible distributions, Cambridge University Press.

[26] Schoutens, W. (2003) Lévy processes in finance, Wiley.

[27] Takeuchi, A., The Bismut-Elworthy-Li type formulae for stochastic differential equations with jumps, Journal of Theoretical Probability, to appear. 

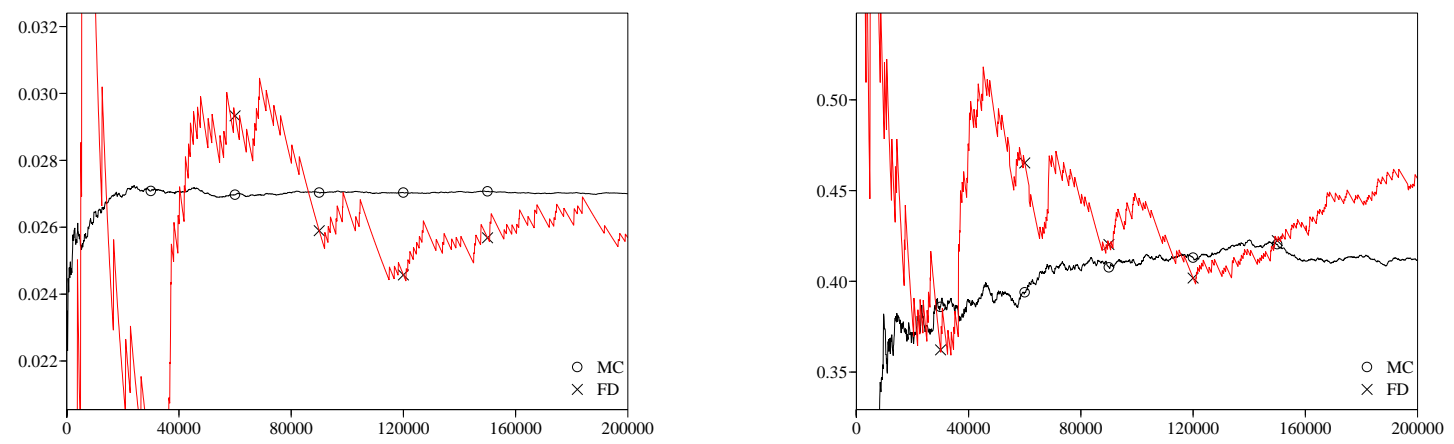

$$
\begin{gathered}
\frac{\partial}{\partial S_{0}} \mathbb{E}\left[e^{-r T} \mathbb{1}\left(S_{T}>K\right)\right] \\
\frac{(\mathrm{FD})}{(\mathrm{MC})}=589 \text { and } \varepsilon=5 e-4 .
\end{gathered}
$$
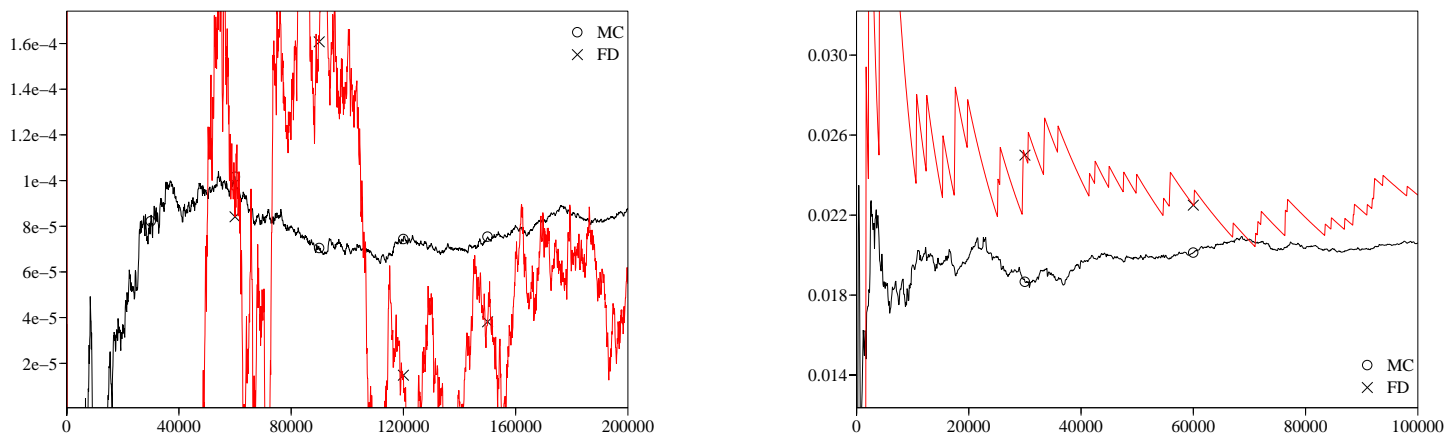

$$
\begin{gathered}
\frac{\partial^{2}}{\partial S_{0}^{2}} \mathbb{E}\left[e^{-r T} \mathbb{1}\left(S_{T}>K\right)\right] \\
\frac{(\mathrm{FD})}{(\mathrm{MC})}=130 \text { and } \varepsilon=2 e-2 .
\end{gathered}
$$

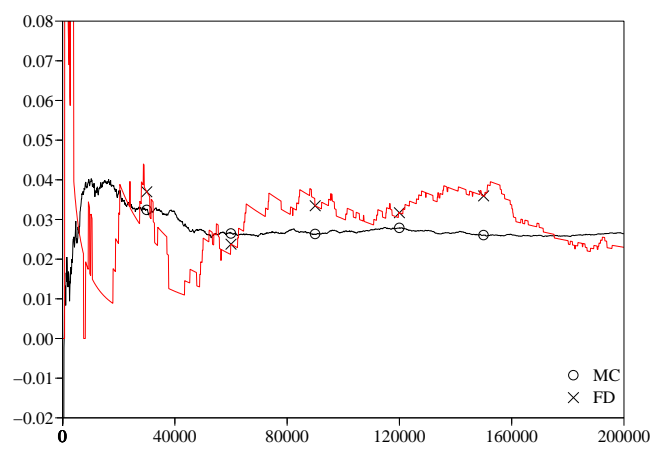

$$
\begin{gathered}
\frac{\partial}{\partial S_{0}} \mathbb{E}\left[e^{-r T} \mathbb{1}\left(\frac{1}{T} \int_{0}^{T} S_{u} d u>K\right)\right] \\
\frac{(\mathrm{FD})}{(\mathrm{MC})}=18 \text { and } \varepsilon=1 e-4
\end{gathered}
$$

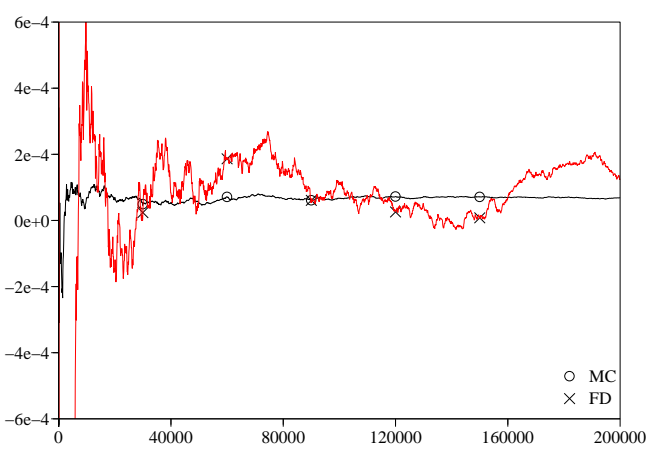

$$
\begin{gathered}
\frac{\partial}{\partial \rho} \mathbb{E}\left[e^{-r T} \mathbb{1}(F>K)\right] \\
\frac{(\mathrm{FD})}{(\mathrm{MC})}=42 \text { and } \varepsilon=1 e-2 .
\end{gathered}
$$

$$
\begin{aligned}
& \frac{\partial^{2}}{\partial S_{0}^{(1)} \partial S_{0}^{(2)}} \mathbb{E}\left[e^{-r T} \mathbb{1}(F>K)\right] \\
& \frac{(\mathrm{FD})}{(\mathrm{MC})}=110 \text { and } \varepsilon=1 e-2 .
\end{aligned}
$$

Figure 1: $\frac{(\mathrm{FD})}{(\mathrm{MC})}$ indicates the variance ratios $\operatorname{Var}(\mathrm{FD}) / \operatorname{Var}(\mathrm{MC})$. The quantity $\varepsilon$ is the increment for the finite difference estimation. 

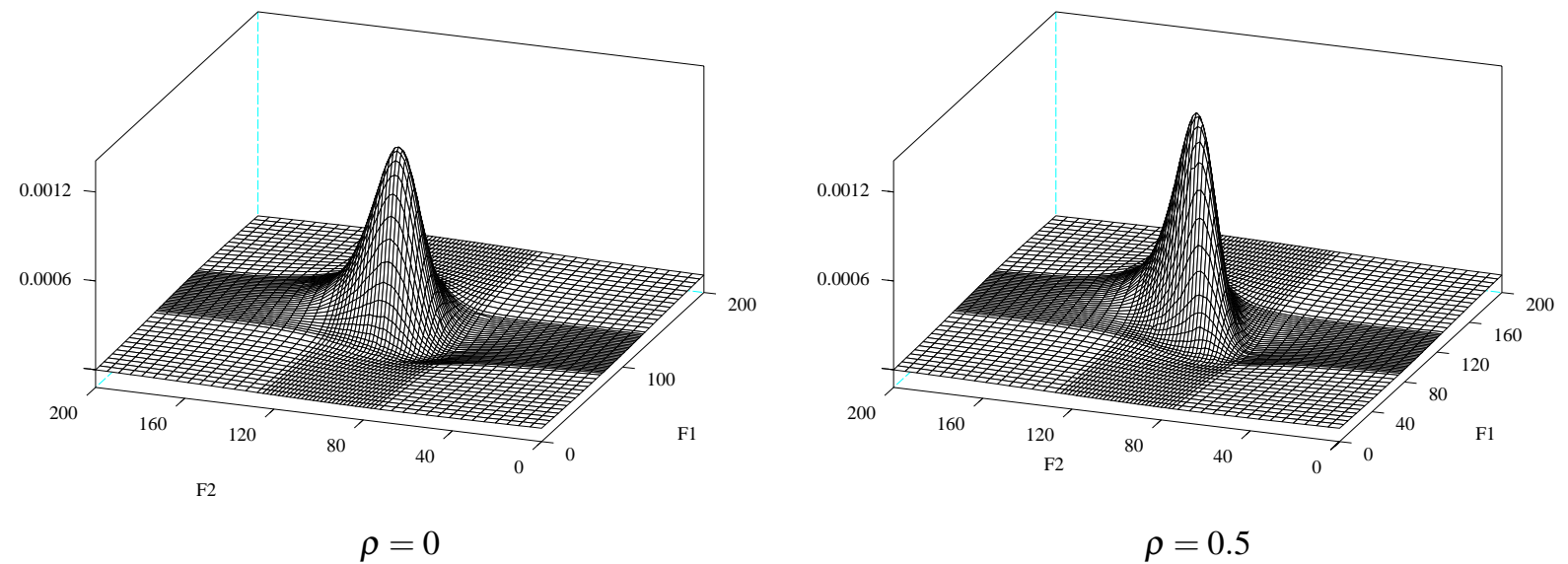

Figure 2: Variance gamma model; parameters are $S_{0}^{(1)}=S_{0}^{(2)}=100, r=0.05, T=1, \sigma_{1}=\sigma_{2}=0.12, \theta_{1}=\theta_{2}=0.1$, and $v=1 / 6$.

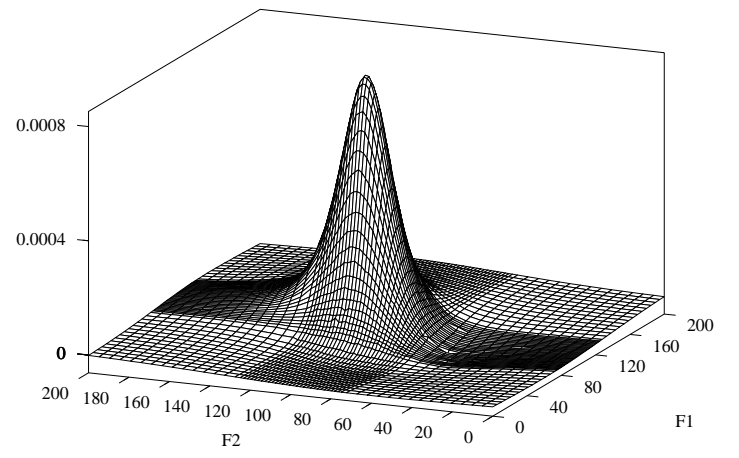

$\rho=0$

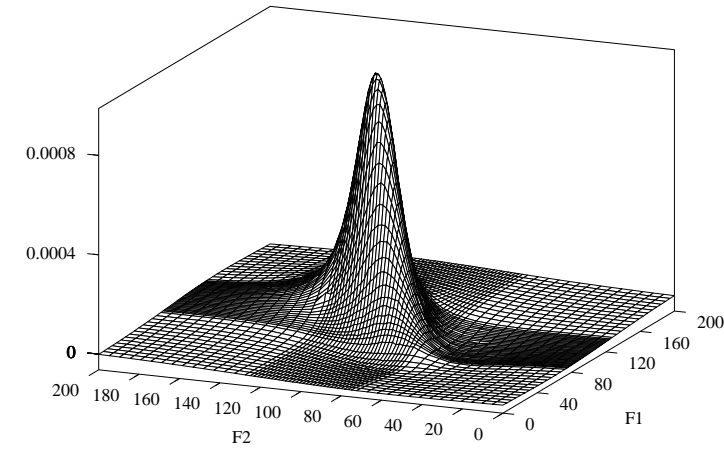

$\rho=0.5$

Figure 3: Normal inverse Gaussian model; parameters are $S_{0}^{(1)}=S_{0}^{(2)}=100, r=0.05, T=1, \alpha=8, \beta=-3, \delta=0.2$. 